\title{
TABLA DE VIDA DEL CUCARRÓN DEPREDADOR Eriopis connexa connexa (Germar) (COLEOPTERA: COCCINELLIDAE)
}

\section{LIFE TABLE OF THE PREDATORY BEETLE Eriopis connexa connexa (Germar) (COLEOPTERA: COCCINELLIDAE)}

\author{
William Duarte Gómez¹, Ingeborg Zenner de Polanía²
}

\begin{abstract}
${ }^{1}$ I.A., M.Sc. Grupo de investigación Fitosanidad. Docente-Investigador, Facultad de Ingeniería Agronómica, Universidad de Ciencias Aplicadas y Ambientales U.D.C.A. Calle 222 No. 55-37, Bogotá, D.C. wduarte@udca.edu.co, ${ }^{2}$ I.A., PhD. Grupo de Investigación Fitosanidad. Docente-Investigador, Facultad de Ingeniería Agronómica, Universidad de Ciencias Aplicadas y Ambientales U.D.C.A. Calle 222 No. 55-37, Bogotá, D.C. izenner@udca.edu.co
\end{abstract}

Rev. U.D.C.A Act. E Div. Cient. 12 (2): 147-155, 2009

\section{RESUMEN}

El conocimiento de los parámetros demográficos de una población de insectos permite determinar sus posibilidades para establecerse y crecer bajo ambientes específicos. Además, proporciona elementos clave para manejar sus crías artificialmente. El objetivo de ese trabajo fue construir una tabla de vida del cucarrón depredador Eriopis connexa, en condiciones de laboratorio. A partir de una cría de laboratorio de este cucarrón alimentado con el áfido Myzus persicae, se observó un fragmento de la población desde el estado de huevo hasta la muerte del estado adulto, en escenarios de $25,5^{\circ} \mathrm{C}$ de temperatura, humedad relativa de 60 a $70 \%$ y fotoperíodo de 12:12. Se registró la mortalidad para cada uno de los estados e instares, la proporción de hembras adultas, el número de huevos puestos por hembra cada cinco días y su fertilidad y la longevidad de los adultos. Con estos datos, se estimaron los siguientes parámetros de la población: tasa de reproducción neta, de 16,07; tiempo generacional, de 30,47 días; tasa intrínseca de crecimiento natural, de 0,072; tasa finita de multiplicación, de 1,08 y tiempo de duplicación, de 9,6 días. Se concluyó que $E$. connexa, bajo las condiciones evaluadas, presentó un alto potencial de establecimiento y crecimiento, lo cual, puede facilitar su papel de controlador biológico de insectos plagas al incorporarse en un agro-ecosistema y, además, ofrece un buen potencial para su cría artificial.

Palabras clave: Control biológico, parámetros demográficos, tasa reproducción, tiempo de duplicación.

\section{SUMMARY}

The knowledge of the demographic parameters of an insect population permits to determine the possibilities of its establishment and growth under specific environment. Furthermore, it provides key elements for the management of its artificial mass rearing. The purpose of this research was to build the life table of the predator Eriopis connexa under laboratory conditions. From a laboratory colony of the coccinellid, fed with the aphid Myzus persicae, a population was observed from egg to adult, under temperature conditions of $25.5^{\circ} \mathrm{C}, 63 \%$ relative humidity and a photoperiod of 12:12. The mortality of each stage and instar was recorded, as well as the proportion of adult females obtained, the number of eggs laid per female every fifth day, its fertility and the adult longevity. With these data the following population parameters were estimated: net reproduction rate of 16.07; generation time of 30.47 days; intrinsic natural growth rate of 0.072 ; finite multiplication rate of 1.08 and duplication time of 9.6 days. It was concluded that the population of 
E. connexa under the studied situations showed a high potential of establishment and growth, facilitating its use as biological control agent of arthropod pests when incorporated in agro-ecosystem and, furthermore, offers a good potential for its artificial rearing.

Key words: Biological control, demographic parameters, reproduction rate, duplication time.

\section{INTRODUCCIÓN}

El cucarrón depredador Eriopis connexa connexa (Germar) (Coleoptera: Coccinellidae) es un insecto muy común en el altiplano Cundiboyacense, tanto en condiciones de campo como bajo invernadero. Sus presas son diversas especies de áfidos (Homoptera: Aphididae), huevos y larvas de primer instar de lepidópteros de varias familias, que se alimentan del follaje y/o de tubérculos de papa y otros insectos de importancia económica (López-Ávila E Espitia-Malagón, 2000; Duarte E Zenner de Polanía, 2009). A pesar de no ser un depredador específico, sino bastante polífago, es considerado un agente promisorio de control biológico, bajo diversas condiciones ambientales.

E. connexa tiene una amplia distribución en América del Sur, hallándose a menudo en forma abundante en Chile, donde se asume que juega un papel significativo en la disminución de las poblaciones de los áfidos Myzus persicae (Sulzer) and Macrosiphum euphorbiae (Thomas) (Quiroz et al. 2005). En cultivos en zonas templadas de Argentina, se ha registrado $E$. connexa como enemigo natural de otros áfidos, $M$. nicotianae Blackman y $M$. persicae (Ohashi \& Urdampilleta, 2003), mientras que en cultivos tropicales de Brasil, se ha detectado su acción controladora sobre poblaciones de algunos lepidópteros, Rachiplusia nu (Gueneé) y Pseudoplusia includens (Walker) (Noctuidae) (Rocha de Moraes et al. 1991) y sobre diversas especies de áfidos (Sarmento et al. 2004). Santos E Pinto (1981), citados por estos últimos autores, destacan su posición prominente entre los coccinellidos, como depredador de áfidos.

Este coccinellido áfido-fago fue inclusive importado a Estados Unidos desde Suramérica, como posible agente controlador de el áfido ruso del trigo, Diuraphis noxia (Mordvilko), como lo afirman Reed E Pike (1991), luego de su viaje exploratorio.
Las tablas de vida, por su gran utilidad, han sido empleadas para comprender los patrones temporales y espaciales de poblaciones de insectos, siendo objetivo de muchas investigaciones que pretenden entender cómo y porqué el número de insectos fluctúa a través del tiempo y el espacio (Peterson et al. 2009).

Una tabla de vida resume, de forma simple, los cambios de una población dentro de una generación, de tal manera que esta información se pueda analizar rápidamente (Varley et al. 1974); asimismo, proporciona una descripción comprensiva de la sobrevivencia, del desarrollo y de la reproducción de una población (Yu et al. 2005). Indica los estados en que una población es más susceptible y también puede revelar los factores de mortalidad más determinantes, suministrando elementos muy útiles en el entendimiento de la dinámica de una población. Cuando se representa en una gráfica, la disminución del número de individuos de una población frente al tiempo, se obtienen curvas de supervivencia, cuya forma proporciona información muy útil acerca de la biología de la plaga (Davis, 1991).

De acuerdo con Southwood (1971), las tablas de vida de "edad específica" son las que mejor se ajustan a las poblaciones de insectos con generaciones discretas, basándose en la mortalidad real de una cohorte, en la cual, los individuos pertenecen a una misma generación. La construcción y el análisis de tablas de vida es uno de los pasos que se deben dar para determinar el impacto de los enemigos naturales sobres sus huéspedes o presas (Bellows et al. 1992).

Yu et al. (2005) mencionan la importancia de obtener el conocimiento de la tabla de vida, tanto del depredador como de la presa, para fines de cría masiva del primero y asegurar su posterior éxito en liberación, en el agroecosistema apropiado. Otros autores afirman que la selección adecuada de un enemigo natural para el manejo de una determinada plaga requiere de la comprensión de su supervivencia, su desarrollo y su reproducción bajo diversas condiciones ambientales, representados y basados en tablas de vida (Atlihan $\mathcal{E}$ Chi, 2008).

La información proporcionada por una tabla de vida de los enemigos naturales de plagas, especialmente, si se ejecuta en diversas condiciones ambientales, o con diversos huéspedes o presas o, si se comparan dos o más especies, como lo realizaron Navarro \& Marcano (2000), permite evaluar el papel del agente de control 
biológico en un sistema plaga-enemigo natural. Resulta igualmente útil para evaluar periódicamente la calidad de una población que se cría de forma artificial o para comparar dos poblaciones de benéficos, mediante el uso del estadístico $r_{m}$ (tasa intrínseca de crecimiento natural). Asimismo, la información proporcionada por una tabla de vida de especies importantes es relevante, con fines de conservación y de manejo integrado de plagas (Naranjo, 2001).

Los parámetros poblacionales son útiles en la caracterización de una población animal, ya que se relacionan con algunos aspectos reguladores del crecimiento de la población. Estas medidas resultan al combinar las funciones de supervivencia y de fecundidad específicos por edades e involucran únicamente al sector hembra de la población. Están incluidos la tasa neta de reproducción, el tiempo generacional, la tasa intrínseca de crecimiento natural y la tasa finita de multiplicación (Rabinovich, 1980).

La tasa de reproducción neta, denotada como $\mathrm{R}_{\mathrm{o}}$, indica el número de progenie hembra producida por cada una en una generación; el tiempo generacional, T, representa el tiempo que un individuo hembra, un huevo, en el caso de los insectos, requiere desde que es puesto hasta que es capaz de dar origen a nueva progenie; la tasa intrínseca de crecimiento natural, simbolizada por $\mathrm{r}_{\mathrm{m}}$, indica la tasa de crecimiento poblacional instantáneo, que mide el número de hembras por hembra por unidad de tiempo y la tasa de multiplicación, conocida como $\lambda$, que se interpreta como el número de individuos que se agrega a la población por individuo y por unidad de tiempo (Rabinovich, 1980). Andrewartha E Birch (1974) definieron la capacidad innata de incremento como la tasa máxima de aumento alcanzada en una combinación particular de temperatura, de humedad y de calidad de alimento, cuando la cantidad de alimento, de espacio y de otros animales de la misma clase, están dentro de un óptimo y otros organismos de clases diferentes son excluidos del experimento.

El objetivo del presente trabajo fue el de construir la tabla de vida del cucarrón depredador, información indispensable para la cría masiva de este insecto en programas de control biológico. De esta manera, se espera implementar un control adicional en el manejo integrado de los cultivos afectados, principalmente, por áfidos y por especies del orden Lepidoptera.

\section{MATERIALES Y MÉTODOS}

Las diferentes evaluaciones se desarrollaron en el laboratorio de entomología de la Universidad de Ciencias Aplicadas y Ambientales U.D.C.A, ubicada en la ciudad de Bogotá D.C., Colombia (448' de latitud norte y $74^{\circ} 03^{\prime}$ de longitud oeste, con una altitud de 2.570msnm).

Adultos de E. connexa fueron colectados en el invernadero de la universidad y trasladados al laboratorio como pie de cría. Especímenes muertos de esta especie fueron depositados en la colección entomológica de la U.D.C.A. Se aparearon y su descendencia se constituyó en la primera generación y su progenie se utilizó para iniciar los diferentes ensayos. Los coccinélidos fueron alimentados con áfidos adultos e inmaduros de las especies M. persicae (Sulzer) y Toxoptera aurantii (Boyer de Fonscolombe) (Homoptera: Aphididae), criados sobre plantas de rosa variedad Manneti y sobre una especie del género Pyracantha (Rosaceae), respectivamente.

La investigación, se realizó en condiciones de 25,5 $\pm 2,5^{\circ} \mathrm{C}$, con una humedad relativa entre 60 y $70 \%$ y con un foto-período 12:12. En recipientes plásticos transparentes, con capacidad de $240 \mathrm{~cm}^{3}$ y con paredes perforadas, se colocaron posturas de diferente número de huevos de $E$. connexa. Al eclosionar éstos con una frecuencia diaria, se realizó la limpieza de los recipientes y se suministró alimento en abundancia. Además, se observó si se presentaban mudas, para trasladar a un nuevo recipiente los individuos recientemente mudados, registrando las fechas y el número de individuos que alcanzaron el siguiente estado. Al momento de obtener los adultos, se separaron por fecha de emergencia en recipientes similares, con el propósito que se aparearan y de poder hacer un seguimiento de su longevidad y su fecundidad. Iniciada la oviposición, diariamente, se sacaron y se contabilizaron los huevos puestos sobre las paredes y tapas de cada caja o en las hojas de rosa provistas con presas. De forma aleatoria, se tomaron algunos de estos huevos para evaluar el porcentaje de eclosión. Al morir, cada uno de los adultos era sacado y depositado en un recipiente marcado con su fecha de emergencia y de muerte, para establecer su duración y para posteriormente hacerle la disección y determinar su sexo; a partir de los datos registrados, se definió la proporción de sexos. Igualmente, cada cinco días y durante toda la vida, se contabilizó la cantidad de huevos 
puesta por cada hembra y la fertilidad de los mismos. Adicionalmente, se estimaron la tasa de oviposición promedio, la tasa de crecimiento reproductivo, la tasa neta de reproducción, la tasa intrínseca de crecimiento natural, el tiempo generacional y el de duplicación y la longevidad de machos y hembras. Finalmente, mediante los procedimientos matemáticos propuestos por Rabinovich (1980), se determinó la supervivencia y la mortalidad de los individuos observados.

\section{RESULTADOS Y DISCUSIÓN}

El valor estimado de la tasa de reproducción neta o tasa de reemplazo $\left(R_{0}\right)$ de $E$. connexa fue de 16,07. Bajo las condiciones del experimento, este valor indica que cada hembra es reemplazada por 16 hembras, al cabo de una generación. Comparativamente, este valor resultó mucho menor que el obtenido por Liu et al. (1997), para otro coccinélido, el Nephaspis oculatus (Blatchley), el depredador de la mosca blanca Bemisia argentifolii Bellows \& Perring (Homoptera: Aleyrodidae), estimado en 54,27 y, todavía, cuatro veces más pequeña que la estimada para Propylaea japonica Thunberg (Coleoptera: Coccinellidae), de 67,6, por Chi $E$ Yang (2003), usando también a M. persicae, como presa. Estas diferencias tan marcadas, por un lado, se pueden atribuir a las diferentes especies, a las condiciones reinantes durante los tres experimentos y al origen geográfico de los insectos benéficos. Asimismo, se interpreta como una capacidad intermedia de crecimiento de la población del depredador bajo estudio, contra las otras dos especies. Frente a los otros dos depredadores, $E$. connexa puede mostrar un incremento de su población más lento y, por lo tanto, no requiere un aumento de los individuos de presas tan alto como sí lo necesitarían las otras dos especies benéficas. Esto representa una ventaja comparativa, pues $E$. connexa no extinguiría a las especies presas, de las cuales se alimenta y, además, esto corresponde a una forma de controlar la densidad de su población, evitando que se incremente, de forma notablemente, superior a la de sus presas.

En cuanto al tiempo generacional $\left(T_{q}\right)$, se estimó un valor de 38,47 días, lo cual, determina que desde que un huevo de $E$. connexa es puesto, se requiere de 38,47 días para que una hembra, proveniente del mismo, inicie su reproducción. Este valor es ligeramente superior al registrado por Chi \& Yang (2003) para P. japonica (37,5 días) y, considerablemente menor que 51,27 días, valor obtenido para N. oculatus, por Liu et al. (1997). Frente a otros coccinellidos, $E$. connexa presenta un tiempo generacional variable a $25,5^{\circ} \mathrm{C}$, pero se considera que esta diferencia no afectaría la capacidad de crecimiento de la población, siempre y cuando el tiempo generacional de sus presas no sea mucho mayor, representando una baja tasa de crecimiento en la población de las presas. Este parámetro es ideal desde el punto de vista de especies plagas, pero desfavorable, ecológicamente, para el establecimiento de este depredador.

La estimación de la tasa intrínseca de crecimiento natural $\left(r_{m}\right)$ proporcionó un valor de 0,072, que significa que la población de $E$. connexa tiene un crecimiento instantáneo positivo cercano al siete por ciento, es decir, que la diferencia entre la tasa de natalidad y la de mortalidad en un tiempo determinado es de esta magnitud. Aunque para poder comparar el valor de este estimador con el de los otros coccinélidos, se debe contar con condiciones idénticas de experimentación. De forma preliminar, se podría decir que la población de $E$. connexa se comporta de manera muy similar a la capacidad de crecimiento de $N$. oculatus, 0,078, reportada por Liu et al. (1997), pero a la vez, es considerablemente inferior a la de $P$. japonica, de 0,11 , que se evalúo a una temperatura y fotoperíodo semejantes, con la misma especie presa y con una humedad relativa de $63 \pm 8 \%$ (Chi $\varepsilon$ Yang, 2003), mientras la HR, en el presente trabajo, varió entre 60 a $70 \%$.

El comportamiento de los parámetros de la tabla de vida es diferente entre depredadores y parásitos; sin embargo, si para $E$. connexa sucediera un comportamiento similar de $r_{m}$ al presentado por Trichogramma pretiosum Riley y T. atopovirilia Oatman E Platner (Hymenoptera: Trichogrammatidae), parasitoides de huevos de Helicoverpa zea (Boddie) (Lepidoptera: Noctuidae), es decir, un aumento de $r_{m}$ al incrementarse la temperatura, sobre la cual se evalúo su desarrollo (Navarro \& Marcano, 2000), se podría pensar que en condiciones de cría cercanas a la temperatura óptima, se tendrían mayores valores de la tasa intrínseca de desarrollo, esto, si se busca el resultado de tener un incremento rápido en la población de laboratorio, con el fin de la liberación en el campo para controlar altos niveles de población de $M$. persicae. Se asume, que lo mismo ocurriría con poblaciones naturales o liberadas del depredador en condiciones con temperaturas cercanas a las de la óptima. Por esta razón, se requiere el desarrollo 
de estudios que examinen el comportamiento de los parámetros de la población estimados en la tabla de vida en las condiciones óptimas de desarrollo (Duarte E Zenner de Polanía, 2009).

Para una cría masiva del depredador, destinada a la liberación contra determinada plaga, sería entonces necesario estimar el valor de $r_{m}$ para cada una de las posibles presas que se piense controlar. De esta manera, se podría entender cómo la interacción entre las dos poblaciones determina el crecimiento de cada una de ellas. Así, presas con valores de tasa intrínseca de crecimiento cercanas o inferiores a 0,07 tendrían una muy rápida reducción en su densidad, especialmente, en ausencia de otras especies de presas. Esto llevaría también a una rápida reducción de la población del depredador, ya sea por muerte o migración, haciendo necesaria su reintroducción al incrementarse, nuevamente, la población de la plaga, a niveles cercanos a los causantes de daño económico. Por otro lado, poblaciones de presas con valores considerablemente superiores a 0,07 podrían garantizar una permanente fuente de alimento para el depredador y favorecerían el desarrollo de sus poblaciones permitiéndoles establecerse y, posiblemente, haciendo innecesaria la cría masiva y la liberación frecuente.

El conocimiento de la tasa intrínseca de otros depredadores que compitan con E. connexa por sus presas en los agroecosistemas donde se podría liberar, al lado de otros parámetros biológicos, como la capacidad de búsqueda y la especificidad, también es un factor determinante que requiere estudios adicionales. Así, se lograría determinar su capacidad competitiva, concluyendo que la especie depredadora que muestra un mayor $r_{m}$, tendría ventajas, por ser la que más rápido podría crecer y aprovechar el recurso presas disponibles.

La tasa finita de multiplicación $(\lambda)$ para la población evaluada de $E$. connexa, se incrementa en 1,08 individuos por día, valor idéntico al obtenido por Liu et al. (1997), para el depredador de la mosca blanca, $N$. oculatus y cercano al de otra especie de coccinélidos, P. japonica (1,119) (Chi \& Yang, 2003). Esta tasa, aparentemente, influenciada tanto por el alimento como por la temperatura, como lo demostraron Tello et al. (2009), para los ácaros depredadores Cydnodromus picanus Ragusa y Phytoseiulus persimilis (Athias-
Henriot) (Acari: Phytoseiidae), alimentados solamente huevos o con la combinación de diversos estados del ácaro Tetranychus cinnabarinus (Boisduval) (Acari: Tetranychidae). Igualmente, resultados similares fueron obtenidos por Perdikis \& Lykouressis (2004) quienes, evaluando al depredador mírido (Hemiptera) Macrolophus pygmaeus Cambur, alimentado con $M$. persicae, observaron una considerable disminución en la tasa finita de multiplicación a medida que aumenta la temperatura de 15 a $25^{\circ} \mathrm{C}$, es decir, si a $E$. connexa se le cambia la presa, como por ejemplo, por la polilla guatemalteca de la papa, Tecia solanivora (Povolny) o alguna plaga de clima medio y se aumenta la temperatura, se podría asumir que su $\lambda$ también cambiaría favorablemente. De allí que para tomar acertadas decisiones, este tipo de parámetros deben ser determinados con las principales presas del depredador y en diversas condiciones climáticas.

El tiempo de duplicación $\left(D_{t}\right)$ que requiere $E$. connexa para doblar su población es de 9,6 días, bajo las condiciones evaluadas. Este lapso es similar al que necesita $N$. oculatus, que corresponde a 8,89 días (Liu et al. 1997) y supera en poco más del $50 \%$ al de $P$. japónica, coccinellido, que requiere apenas 6,17 días (Chi \& Yang, 2003).

Comparando los datos de la tabla de vida de $P$. japonica, aunque este coccinélido no está registrado en Colombia, con los parámetros señalados por este estudio, se observa que el primero posee características más favorables para el crecimiento de su población en condiciones semejantes a las evaluadas para $E$. connexa y teniendo a $M$. persicae como presa. No obstante, no se recomienda su introducción, ya que es mejor aprovechar los beneficios naturales de una especie ya adaptada, que introducir a nuestras condiciones especies foráneas no adaptadas.

La figura 1 representa la proporción de individuos del depredador que pasa al siguiente estado o instar larval. Allí, se observa que la menor supervivencia ocurrió en el estado de huevo, ya que solo el $70 \%$ de los huevos, con los cuales se inició el ensayo, comenzaron el estado de larva, en contraste con el $25 \%$ de los individuos que inició el estado de larva y que llegó a formar la pupa. Dentro del estado de larva, la menor supervivencia se presentó entre el primer y segundo instar, cuando la mortalidad fue del $12 \%$, mostrando que a $25,5^{\circ} \mathrm{C}$ el inicio de la 
fase inmadura de $E$. connexa es la más susceptible y, que a partir del tercer instar, la mortalidad fue muy baja; sin embargo, la supervivencia total de huevo a adulto no alcanzó el 50\% (Figura 1), lo cual, es considerado desventajoso en comparación con otros coccinélidos que logran una supervivencia del 70\% (Brown et al. 2003).
La figura 2 muestra la supervivencia de las hembras $\left(l_{x}\right)$ y su fecundidad $\left(m_{x}\right)$. Llama la atención el hecho de la alta mortalidad (cerca del 40\%) en los primeros 15 días de vida, los cuales, coinciden con la fase preimaginal. Posteriormente, se observó otra fuerte caída en la supervivencia del $25 \%$, aproximadamente, entre los 20 y 33 días de vida, los que incluyen el tiempo de

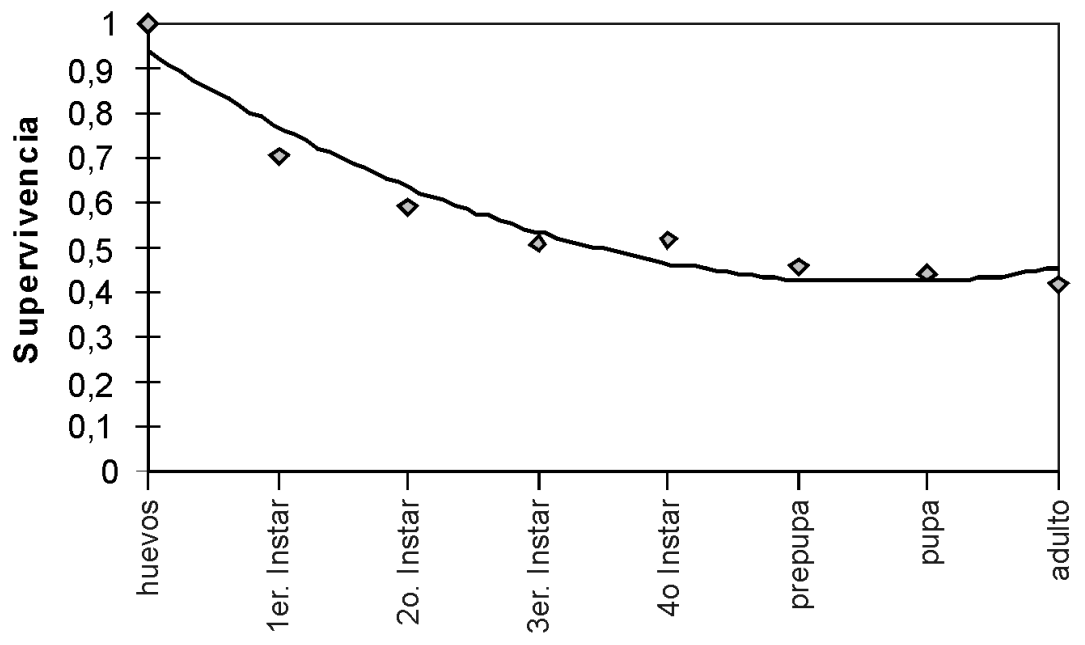

Estados de desarrollo

Figura 1. Curva de supervivencia de Eriopis connexa connexa a $25,5^{\circ} \mathrm{C}$.

preoviposición y el inicio de la oviposición. La máxima fecundidad se alcanzó a una edad de 37,5 días, en que las hembras llegaron a originar cerca de diez nuevas hembras en promedio, por cada período de cinco días. Para esta edad, la supervivencia se redujo a un 33\%, lo que quiere decir, que de cada 100 huevos hembras, solo 33 llegarán en promedio a alcanzar la edad de su máxima fecundidad.

De otra parte, la curva de fecundidad de hembras indica que existe un comportamiento cíclico y con tendencia descendente, con picos a los 55, 75 y 85 días, con fecundidades de 6,3; 5,3; 2,4 y 2 hembras por hembra en cada período de cinco días, respectivamente, hasta los 105 días, cuando la fecundidad se hace nula. Finalmente, se observó que muy pocas hembras llegan a ser muy longevas, como la que alcanzó los 168 días de edad.

En el presente estudio además se encontró en las condiciones de la evaluación, como se puede observar en la figura 3, que cada hembra puede llegar a ovipositar un promedio de 112,65 huevos durante su ciclo de vida, distribuidos en once posturas, con un promedio de diez huevos por postura. El período de mayor oviposición, se ubica entre los 27,5 y 72,5 días de edad de las hembras, con picos de oviposición a los 37,5 (15\% del total de huevos) y 57,5 días ( $10 \%$ del total de los huevos). Además, se observó que la mitad de los huevos se ovipositó entre los 22,5 y 47,5 días y, que el $50 \%$ restante, se distribuyó en el período comprendido entre los 47,5 y 97,5 días de edad de las hembras. Esto indica que hay una mayor concentración de la oviposición en los primeros 25 días de la fase reproductiva. En los resultados también se encontró que el 95\% de los huevos se ovipositó a los 50 días de emergida la hembra adulta (Figura 3), lo que significa que en condiciones similares a las utilizadas para esta evaluación y a pesar de la longevidad de algunas hembras, no se justifica mantenerlas más allá de los 50 días; se pueden desechar de las crías o liberar en el campo por no ser productivas, ya que al mantenerlas consumen alimento y requieren labores de manejo. 

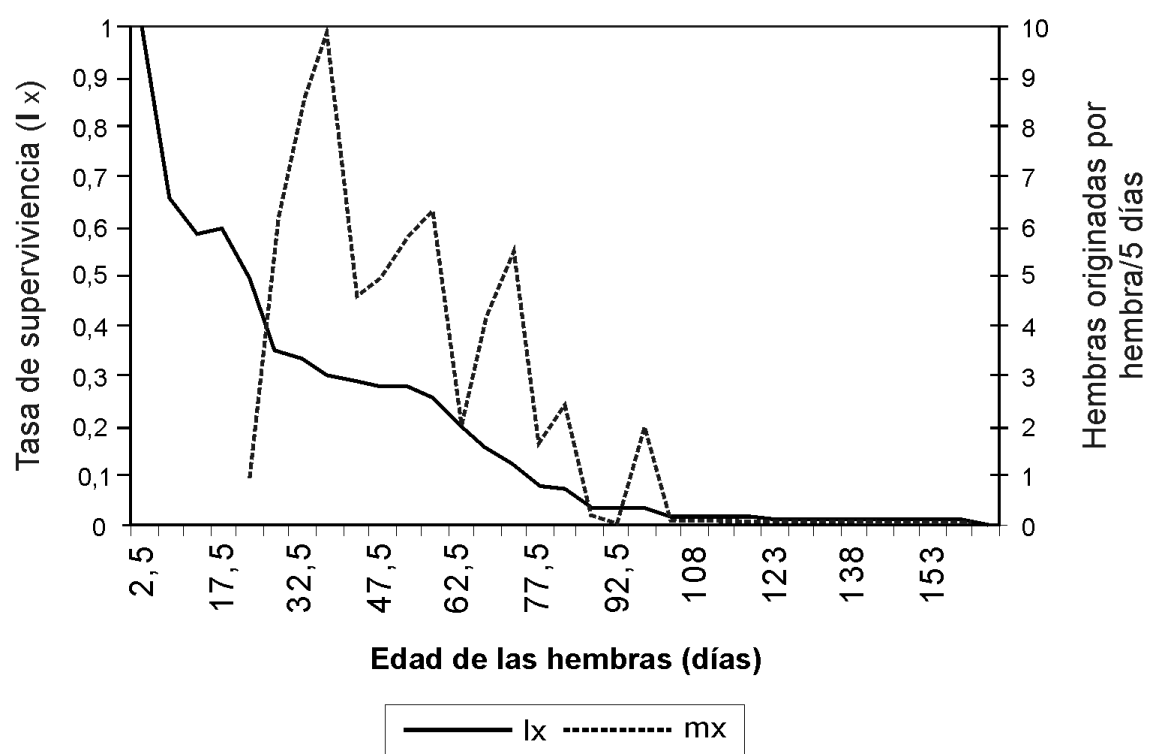

Figura 2. Curvas de supervivencia $\left(l_{x}\right)$ y fecundidad $\left(m_{x}\right)$ de hembras de Eriopis connexa connexa.

En relación con la liberación de Eriopis connexa y lo que esto puede significar a nivel de campo, se debe considerar y evaluar la posibilidad de su establecimiento prolongado en un agro-ecosistema, iniciando esta labor con la liberación de hembras adultas y de larvas en épocas de alta densidad de presas, con el fin de facilitar este propósito; sin embargo, si la finalidad es controlar una alta densidad de una especie presa, se deben liberar los estados más voraces en cantidad suficiente para disminuir rápidamente la población de la plaga. En general, se concluye que el cucarrón depredador, Eriopis connexa connexa representa un buen candidato para establecer controles biológicos, ante todo de áfidos, ya sea con altas densidades de presas o al iniciarse una

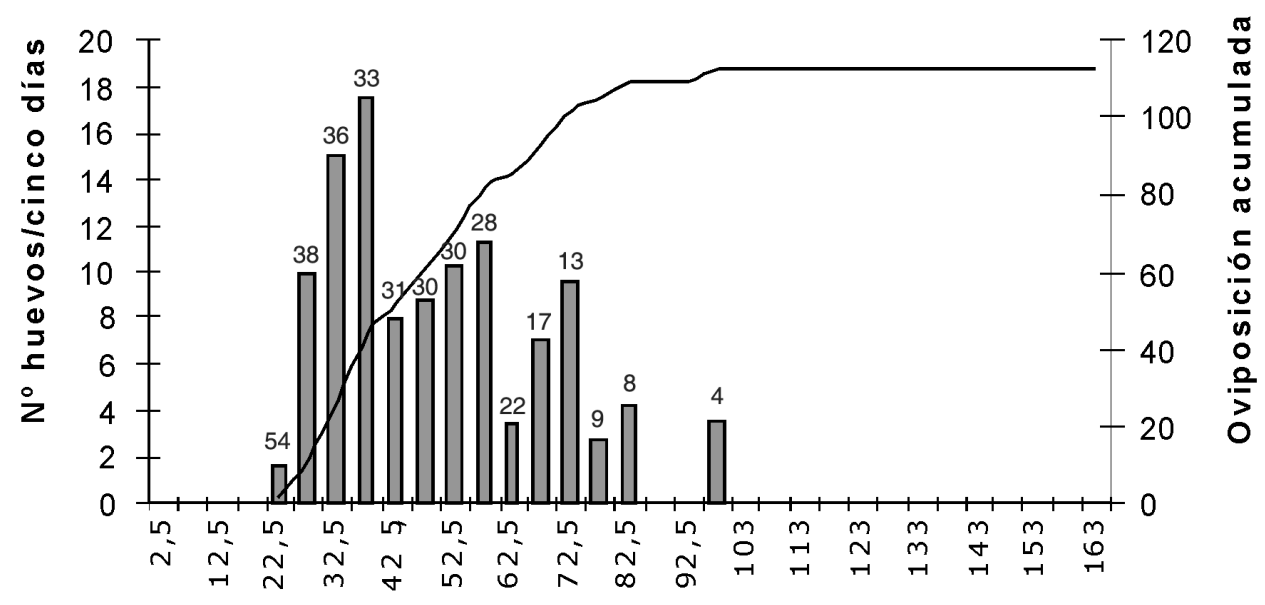

$\square$ No. Huevos/hembra $\quad$ Oviposición acumulada

Figura 3. Relación entre la oviposición en periodos de cinco días y la oviposición acumulada de Eriopis connexa connexa. Los números sobre las barras indican el número de hembras observadas en cada rango de edad. 
infestación, que pueda llegar a niveles de advertencia económica.

Se recomienda continuar los estudios encaminados a establecer las condiciones más apropiadas de cría, incluyendo dietas merídicas y enfocando los esfuerzos hacia generar conocimiento útil en el control de las plagas, económicamente más importante, ante todo, en cultivos de plantas ornamentales.

AGRADECIMIENTOS: Los autores hacen manifiesta su reconocimiento a la U.D.C.A y a sus directivas por el apoyo y la financiación. Conflicto de intereses: Hacemos constar que el manuscrito fue preparado y revisado con la participación de los dos autores, quienes declaramos que no existe ningún conflicto de intereses que ponga en riesgo la validez de los resultados presentados.

\section{BIBLIOGRAFÍA}

1. ANDREWARTHA, H.G.; BIRCH, L.C. 1974. The Distribution and Abundance of Animals. The University of Chicago Press. Chicago and London. 782p.

2. ATLIHAN, R.; CHI, H. 2008. Temperature-dependent development and demography of Scymnus subvillosus (Coleoptera: Coccinellidae) reared on Hyalopterus pruni (Homoptera: Aphididae). J. Econ. Entomol. 101(2):325-333.

3. BROWN, G.C.; SHARKEY, M.J.; JOHNSON, D.W. 2003. Bionomics of Scymnus (Pullus) louisianae J.Chapin (Coleoptera: Coccinellidae) as a predator of the soybean aphid Aphis glycines Matsumura (Homoptera: Aphididae). J. Econ. Entomol. 96(1):21-24.

4. BELLOWS, T.S.; DRIESCHE, R.G.; ELKINTON, J.S. 1992. Life table construction and analysis in the evaluation of natural enemies. Ann. Rev. Entomol. 37:587-614.

5. CHI, H.; YANG, T.CH. 2003. Two-Sex life table and predation rate of Popylaea japonica Thunberg (Coleoptera: Coccinellidae) fed on Myzus pesicae (Sulzer) (Homoptra: Aphididae). Environ. Entomol. 32(2):327-333.
6. DAVIS, R.G. 1991. Introducción a la Entomología. Ed. Mundiprensa, Madrid. 352p.

7. DUARTE G., W.; ZENNER DE POLANÍA, I. 2009. Efecto de la temperatura sobre el desarrollo de Eriopis connexa connexa (Germar) (Coleoptera: Coccinellidae). Rev. U.D.C.A Act. \& Div. Cient. 12(2): 135-145.

8. LIU, T-X.; STANSLY, P.A.; HOELMER, K.A.; OSBORNE, L.S. 1997. Life History of Nephaspis oculatus (Coleoptera: Coccinellidae), a Predator of Bemisia argentifolii (Homoptera: Aleyrodidae). Ann. Entomol. Soc. Am. 90(6):776-782.

9. LÓPEZ-ÁVILA, A.; ESPITIA-MALAGÓN, E. 2000. Plagas y benéficos en el cultivo de la papa en Colombia. Boletín Técnico Divulgativo. MIP. Corpoica-PRONATTA. Produmedios. Bogotá D.C. 35p.

10. NARANJO, S.E. 2001. Conservation and evaluation of natural enemies in IPM systems for Bemisia tabaci. Crop Protection. 20: 835-852.

11. NAVARRO, R.V.; MARCANO, R. 2000. Tablas de Vida de Trichogramma pretiosum Riley y T. atopovirila Oatman y Platner en laboratiorio. Agronomía Tropical (Venezuela). 50(1):123-134.

12. OHASHI, D.V.; URDAMPILLETA, J.D. 2003. Interacción entre insectos perjudiciales y benéficos en el cultivo del tabaco de Misiones, Argentina. Rev. Invest. Agrícolas. 32(2):113-124.

13. PERDIKIS, D.Ch.; LYKOURESSIS, D.P. 2004. Myzus persicae (Homoptera: Aphididae) as suitable prey for Macrolophus pygmaeus (Hemiptera: Miridae) population increase on pepper plants. Environ. Entomol. 33(3):499-505.

14. PETERSON, R.K.D.; DAVIS, R.; HIGLEY, L.G.; FERNANDES, O.A. 2009. Mortality risk in insects. Environ. Entomol. 38(1):2-10.

15. QUIROZ E., C.; LARRAÍN S., P.; SEPÚLVEDA R.P. 2005. Abundancia estacional de insectos vectores de virosis en dos ecosistemas de pimiento (Capsicum annum L.) de la región de Coquimbo, Chile. Agric. Técnica. 65(1):3-19. 
16. RABINOVICH, J.E. 1980. Introducción a la Ecología de Poblaciones Animales. Ed. CECSA. Caracacas (Venezuela). 313p.

17. REED, D.K.; PIKE, K.S. 1991. Summary of an exploration trip to South America. International Organization for Biological Control, Nearctic Regional Section Newsletter. 36:16-17.

18. ROCHA DE MORAES, R.; LEK, A.E.; BELARMINO, E.C. 1991. Inimigos naturais de Rachiplusia nu (Guenée, 1852) e Pseudoplusia includens (Walter, 1857) (Lepidoptera: Noctuidae). Pesq. Agropec. Bras. 26(1):57-64.

19. SARMENTO, R.A.; GOMES DE OLIVEIRA, H.; HOLTZ, A.M.; MARQUES DA SILVA, S.; SERRÃO, J.E.; PALLINI, A. 2004. Fat body morphology of Eriopis connexa (Coleoptera, Coccinelidae) in function of two alimentary sources. Braz. Arch. Biol. Technol. 47(3):407-411.

20. SOUTHWOOD, T.R.E. 1971. Ecological Methods, with particular reference to the study of Insect populations. Ed. Chapman and Hall Ltd. London. 391p.
21. TELLO, V.; VARGAS, R.; ARAYA, J.; CARDEMIL, A. 2009. Biological parameters of Cydnodromus picanus and Phytoseiulus persimilis raised on the carmine spider mite, Tetranychus cinnabarinus (Acari: Phytoseiidae, Tetranychidae). Cien. Inv. Agr. (Chile). 36(2):277-290.

22. VARLEY, G.C.; GRADWELL, G.C.; HASSELL, M.P. 1974. Insect Population Ecology, an Analitical Approach. U. California Press. 212p.

23. YU, J-Z.; CHI, H.; HUEI CHEN, B-H. 2005. Life table and predation of Lemnia biplagiata (Coleoptera: Coccinellidae) fed on Aphis gossypii (Homoptera: Aphididae) with a proof on relationship among gross reproduction rate, net reproduction rate, and preadult survivorship. Ann. Entomol. Soc. Am. 98(4):475-482.

Recibido: Agosto 9 de 2009

Aceptado: Noviembre 8 de 2009 